Eine Erweiterung der Methode muB hier noch kurz besprochen werden, nämlich die sogenannte partielle und totale Korrelation (wie Yule sie nennt). Wenn. wir wissen, daB $X$ nicht allein von $Y$, sondern auch von $Z, U, V$ usw. abhängt, wo $Z, U, V$ usw. andere variable Größen sind, so kann man $X$ berechnen, indem man die einzelnen Abhängigkeiten von $Y$, $Z, \quad U$ usw. zusammensetzt und schreibt $x=$ $a y+b z+c u+d v+\ldots$, wo wie früher die kleinen Buchstaben die Abweichungen von den Mittelwerten der Reihen bezeichnen. Man kann die par tielle Korrelation von $x$ mit $y$, von $x$ mit $z$ usw. berechnen, man kann aber auch ausdrücken, bis zu welchem AusmaBe $x$ von allen bekannten Variabeln $y$, $z, u$ usw. zusammen abhängt, und nennt dieses Ausmaß den totalen Korrelationsfaktor $m$. Es ist z. B. für bloß 3 Variable

$$
m=\sqrt{\frac{r_{2}^{2}+r_{3}^{2}-2 r_{1} r_{2} r_{3}}{1-r_{1}^{2}}}
$$

wo $r_{1}$ den Korrelationsfaktor von $y$ und $z$, $r_{2}$ von $x$ und $z, r_{3}$ von $x$ und $y$ bedeutet.

Der große Vorteil dieser Methode ist, daß wix mit bloß statistischen Daten die zu suchende Größe in Form einer Gleichung durch die bekannten Gröben ausdrücken können, ohne irgend etwas über den Mechanismus des Zusammenhangs derselben zu wissen. Die Gleichung wird freilich stets nur angenähert gelten, aber man kann offenbar die Annäherung steigern, wenn es gelingt, neue Variable aufzufinden und der Korrelation anzureihen, welche von EinfluB auf die untersuchte GröBe sind. Es bodeutet diese Methode also einen ganz wesentlichen Fortschritt in der Ausnutzung statistischer Daten.

Als Beispiele für diese zusammengesetzten Korrelationen führen wir hier meteorologische Studien des Direktors der Observatorien von Britisch-Indien, Gilbert T. Walker, an (Mem. of the Indian Met. Department Vol. XXI, II, 1910), aus denen man zugleich ersieht, wie die Methode praktische Wichtigkeit erlangt hat, indem hier Größen zueinander in Beziehung gesetzt sind, die nicht gleichzeitig, sondern nacheinander auftreten. Es besteht offenbar kein Hindernis, die Korrelationsmethode auch auf solche Fälle anzuwenden, sobald eben eine Korrelation dieser Art existiert. Man erhält dadurch die Möglichkeit, gewisse GröBen roraus zu berechnen, cine Prognose auf statistischer Grundlage aufzustellen. Es ist bezeichnend, daB sich die Methode an Gegenständen von äußerster praktischer Wichtigkeit entwickelt hat; und zwar stellte sich Walker die Aufgabe, die Methode zur Voraussage der Regenmengen in Indien zu verwenden, welche für die nächste Ernte in Indien und damit für die Frage der Hungersnöte von größter Bedeutung sind. Ein ähnlicher Fall, den er gleichfalls behandelt, ist die Höhe der Nilflut, die wieder für Bewässerung und Ernte Ägyptens ausschlaggebend ist.

Die Untersuchung liefert zunächst für den indischen Monsunregenfall eine Abhängigkeit von der vorhergegangenen Schneehöhe im Himalaya, dann von dem Luftdruck auf Mauritius und jenem in Südamerika gewisse Zeit vorher, endlich auch von dem vorhergegangenen Regenfall in Sansibar. Durch Berechnung der einzelnen partiellen. Korrelationsfaktoren ergibt sich eine eigentümliche Gleichung, in welcher der Monsunregenfall als lineare Funktion all dieser vier Faktoren dargestellt wird, so dab man - freilich nur mit einiger Wahrscheinlichkeit - den zu erwartenden Regenfall aus den vier beobachteten Größen voraus berechnen kann. In ähnlicher Weise wird die Nifflut dargestellt.

Heutzutage, wo selbst in den exakten Naturwissenschaften, wie der Physik, die statistische Auffassung der Ereignisse immer mehr um sich greift, ist es gewil sehr zu begrüBen, daß die Methoden der Statistik weiter ausgebildet werden. Um so mehr wäre es zu wünschen, daB die Anwendung derselben auch in der deutschen Literatur weitere Fortschritte machte. Es ergibt sich an vielen Orten die Frage, wie weit eine Regel durch Unkenntnis der einfluBnehmenden Faktoren, wie weit durch bloßen Zufall zustande kommt (vergl. die Inaugurationsrede von Franz Exner, Wien 1909), wie es etwa in der Vererbungslehre (Mendelsche Regeln) der Fall zu sein scheint. Sind z. B. die meteorologischen Erscheinungen zum reil Ergebnisse zufälliger Ereignisse und als solche nicht oder nur angenähert vorhersehbar? Auf solche Fragen wird eine erweiterte Beschäftigung mit den Ergebnissen der Statistik hoffentlich in einiger Zeit Licht werfen.

\section{Neuere Bestrebungen auf dem Gebiete der Tuberkulosebehandlung.}

Von Prof. Dr. Carl Bruck, Breslau.

Wenn wir von Tuberkulosebehandlung sprechen, so müssen wir uns bewußt bleiben, daß diejenigen Verfahren, die wir bisher unter diesem Begriffe verstehen, eher eine Behandlung Tuberlulöser als eine Therapie der Tuberkulose darstellen. Denn trotz der völligen ätiologischen Klärung der Tuber kulose sind die üblichen Heilverfahren bei dieser Infektionskrankheit von dem idealen Ziele noch recht weit entfernt. Das Ideal der Therapie bei einer Krankheit mit bekanntem Erreger muß naturgemäf das sein, die Krankheitsursache zu beseitigen, d. h. ohne Schädigung des infizierten Körpers die krankmachenden Mikroorganismen zu vernichten. Wenn wir unsere heutigen therapeutischen Maßnahmen bei Tuberkulose betrachten, so schweift der Blick von den Sanatorien der Höhenluftkurorte, den Plätzen des sonnigen Südens nach den Heil- und Kuranstalten der Heimat. Ửberall sehen wir dasselbe Prinzip: Luft, Licht und Sonne. Unter möglichster seelischer und körperlicher Ruhe wird versucht, den kranken Körper für den Kampf mit den Bazillen zu stählen, durch physikalische MaBnahmen (hydrotherapeutische Prozeduren, Atemgymnastik usw.) den Organismus oder das kranke Organ zu stärken, eventuell durch gewisse Medikamente, denen erfahrungsgemäb eine gewisse Wirkung zugeschrieben wird (Inhalationen, Lebertran, Kreosot usw.), den Prozeß zu becinflussen. 
Oder wir sehen, wie bei gewissen Formen der Tuberkulose (Drüsen-, Gelenkerkrankungen) entweder das Krankhafte durch die Kunst des Chirurgen beseitigt wird oder wie wiederum dureh physikalische Maßnahmen die Abwehrkräfte des Organismus in Anspruch genommen werden (Hyperämiebehandlung). Der Dermatologe endlich trach tet bei der Tuberkulose der Haut, dem Lupus, den Krankheitsherd durch operative Eingriffe, durch lokale chemisch oder physikalisch-chemisch wirkende Prozeduren (Atzungen, Lichtbehandlung) zu entfernen oder zu zerstören, in der Voraussetzung, daß nach der Beseitigung oder bei der Abheilung des Krankheitsproduktes auch eine Schädigung oder eine Abtötung der krankheitserregenden Bazillen erfolgt.

So grol die Erfolge der mit den angedeuteten Verfahren erzielten Resultate unzweifelhaft sind, wie enorme Fortschritte auf dem Gebiete der Tuberkulosetherapie auch gemacht wurden, so darf man sich doch nicht verhehlen, daB der Arzt dem Tuberkulösen gegenüber heute noch immer die Rolle eines Gärtners spielen muB, der einen Baum, dessen Wipfel von kraftsaugenden Parasiten durchsetzt sind, dadurch heilen will, daß er seiner Wurzel die günstigsten Nährbedingungen schafft, seinem Stamme jede Schädlichkeit fern hält oder der einen unter dem Einfluß des Schmarotzers zugrunde gehenden oder bereits vernichteten Ast abschneidet.

Unter diesen Umständen erregte es bekanntlich enormes Aufsehen, als wir zum ersten Male durch $R$. Koch im Tuberkulin ein spezifisches Mittel kennen lernten, d. h. eine Substanz, die auf dem Blutwege eine ganz spezielle Wirkung auf die durch die Tuberkelbazillen gesetzten Krankheitsherde ausiibt. Leider ist der anfänglichen Begeisterung eine starke Enttäuschung gefolgt, die sehr bald in eine ganz ungerechtfertigte Verurteilung des Tuberkulins überging, bis man erst allmählich im Laufe der Zeit gelernt hat, den eigentlichen und, wie hervorgehoben werden muB, außerordentlich groken Wert des Tuberkulins für die Diagnose und auch für die Therapie richtig einzuschätzen. Das muß allerdings heute zugegeben werden, daß das Tuberkulin ein "spezifisches" Mittel im idealen Sinne nicht ist, denn die Spezifizität richtet sich lediglich auf das Krankheitsprodukt, nicht auf den Krankheitserreger. Wenn wir also zu Heilzwecken Tuberkulin anwenden, dürfen wir nicht erwarten, daß wir durch die Injektionen die Tuberkelbazillen direkt schwächen oder abtöten, sondern das, was wir erreichen, ist eine entzündliche Reaktion des von Tuberkelbazillen durchsetzten kranken Gewebes, ein Vorgang, der in einen Heileffekt übergehen kann und ähnlich ist demjenigen, wie er sich Z. B. an einem mit Hyperämie behandelten Gelenk oder an einem mit Lichtstrahlen zur Entzündung gebrachten Lupusherd abspielt. Es kann somit die Tuberkulinwirkung nicht etwa verglichen werden mit der direkt gegen den Erreger gerichteten Wirkung gewisser spezifischer Medikamente (ChininMalaria, $\mathrm{Hg}$, Salvarsan-Lues).

Ich möchte hier nicht die etwaigen immunisatorischen bzw. giftvernichtenden Eigenschaften des Tuberkulins auseinandersetzen, sondern zur Erklärung der eigenartigen Tuberkulinwirkung nur die Theorie kurz skizzieren, die Wassermann und ich aufgestellt and durch Experimente belegt haben, ohne auf die Einwände, die unseren Anschauungen entgegengehalten worden sind und die zahlreichen anderen Theorien einzugehen. - Die merkwürdige Tatsache, daß nach minimalen, entfernt vom Krankheitsprodukt injizierten Dosen Tuberkulin an dem erkrankten Herd eine Reaktion auftreten kann, dals also z. B. ein Lupusherd sich entzündlich rötet, ein Lungenherd vermehrte physilalische Symptome, gesteigerten Auswurf zeigt, erklärt sich nach unseren Versuchen damit, daB im Krankheitsherd ein experimentell nachweisbarer Gegenkörper, das Antituberkulin, gebildet wird, der kraft seiner spezifischen chemischen Affinität das Tuberkulin aus der Blutbahn abfängt und in den Herd konzentriert. Bei der Bindung des Tuberkulins und Antituberkulins kommt es nun weiter zur Verankerung eines natürlichen, dem Körper zur Verfügung stehenden und aus den weißen Blutkörperchen stammenden fermentativ wirkenden Schutz- und Heilstoffes, des sog. Komplementes, dessen Wirkung zugleich mit seinen Mutterzellen, den Leukocyten, in Gestalt jener entzündlichen Reaktion in Erscheinung tritt, der wiederum eine heilsame Einschmelzung des kranken Gewebes folgt.

Ich habe diese unsere Antituberkulintheorie hier nur angedeutet, um zu zeigen, dab die Heilwirkung des Tuberkulins sich in erster Linie auf das Gewebe und nicht anf den Mikroorganismus richtet und daß, wenn bei diesen Heilungsvorgängen auch allmählich Tuberkelbazillen zugrunde gehen, dies nur einen sekundären Vorgang darstellt. - Wenn also somit die Tuberkulintherapie keine direkt ätiologische Methode im reinsten Sinne ist, so muB man doch sagen, daß das Tuberkulin ganz abgesehen von seiner unersetzlichen diagnostischen Bedeutung - richtig und vorsichtig angewendet, als Heilmittel mit an erster Stelle steht, und daß die vielfach gleichzeitig mit der physikalisch-diätetischen Behandlung durchgeführten Tuberkulinkuren ihre volle Berechtigung und Bedeutung haben.

Die zahlreichen Versuche auf dem Wege der aktiven und passiven Immunisierung, d. h. durch Behandlung mit abgetöteten Tuberkelbazillen oder durch Heilseren zu einem direkten und für den Körper unschädlichen Abtötungsverfabren für den Tuberkelbazillus zu gelangen, müssen bisher als wenig aussichtsreich bezeichnet werden. Wenn auch über Erfolge mit den verschiedensten Präparaten berichtet worden ist, so hat sich doch keines eine allgemeine Anerkennung $\mathrm{zu}$ erringen vermocht. Verheißender scheint der in allerjüngster Zeit unternommene Versuch zu sein, durch Vaccination, d. h. durch Behandlung mit abgeschwächten, avirulenten aber noch lebenden Tuberkelbazillen einen Erfolg zu erzielen. Wenigstens klingen die Berichte von Friedmann, der mit aviruIenten, von Schildkrötentuberkulose gezüchteten Bazillen behandelt, vielversprechend. 
Bei den wie gesehen immerhin bescheidenen Aussichten, welche die Immunotherapie bei Tuberkulose bietet, erscheint es nicht verwunderlich, daß man sich angesichts der grobartigen Erfolge, welche in neuerer Zeit die von Ehrlich begründete Chemotherapie bei anderen Infektionskrankheiten erzielt hat, auch bei der Tuberkuloseforschung jenen Methoden zugewandt hat. Die Aufgabe der Chemotherapie ist es, durch methodische Untersuchungen chemische Substanzen ausfindig zu machen, die im Tierkörper eine spezifische abtötende Wirkung auf bestimmte Mikroorganismen oder eine Gruppe von solchen ausüben. Die Schwierigkeit beruht darin, zu solchen Stoffen zu gelangen, die einerseits die Mikroorganismen im 'Tierkörper direkt aufsuchen, um ihnen ihre Wirkung mitzuteilen (Parasitotropie) und anderseits cine geringe Affinität zu den tierischen $\mathrm{Or}_{\mathrm{r}}$ ganen haben, die zu einer Vergiftung des Körpers führen kann (Organotropie). Da es nicht immer möglich ist, durch Veränderungen der chemischen Konstitution Substanzen an den Krankheitsherd bzw. den Erreger heranzubringen, hat man sich auch dadurch geholfen, daß man sich für den Transport des betreffenden Mittels sog. Leitschienen bediente. So hat $v$. Wassermann dureh Kuppelung des krebszellen-schädigenden Selens an bestimmte Farbstoffe eine therapeutische Wirkung erzielt. -

Was die Tuberkulose betrifft, so war schon vor Jahren von $A$. Neißer auf die Möglichkeit hingewiesen worden, mit Milfe des spezifisch den Krankheitsherd aufsuchenden Tuberkulins gewisse Medikamente, von denen man sich eine Wirkung auf die Tuberkelbazillen versprach, an den gewünschten Ort zu leiten.

In der Tat hat man nun zuerst in Verfolg der Ehrlichschen und Wassermannschen Versuche eine Substanz zum Gegenstand der Untersuchung gemacht, von der eine Einwirkung auf tuberkulöse Prozesse bekannt ist, das Jod. - So haben Bauer und Murschhauser, Kapsenberg und Sternberg Jodtuberkuline hergestellt und eine therapeutische Wirkung gesehen.

Ws haben ferner Herxheimer und A7tmann sowie Bernhardt über einen Heileffekt bei Hauttuberkulose berichtet, der dureh eine Kombination von Salvarsaninfusionen mit Tuberkulininjektionen erzielt wurde.

Chemotherapeutische Versuche sind dann weiter auf Veranlassung von Finckler mit Jodmethylenblau und Kupferverbindungen gemacht worden. Mit diesen Präparaten wurden von Gräfin Linden Heilungen bei Meerschweinchen-Tuberkulose gesehen, Meifner berichtet über Besserungen bei Lungentuberkulose und Strauß über auffallende Beeinflussungen des Lupus. Da aber meines Erachtens die als besonders bedeutungsvoll erscheinenden Erfolge von $B \operatorname{trau} \beta$ aus hier nicht näher zu erörternden Gründen nicht beweisend sind, so muB abgewartet werden, inwieweit jene Substanzen vom Blutwege aus beim Menschen eine Wirkung entfalten werden.
Ich selbst habe in Verfolg eigener früherer chemotherapeutischer Versuche bei Tuberkulose eine Substanz studiert, die bisher noch nie therapeutisch angewendet worden war, das Aurum kaliumeyanatum.

Im Jahre 1890 hat $R$. Koch mitgeteilt, daß von allen von ihm in vitro untersuchten Präparaten die Cyangoldverbindungen die stärkste desinfektorische Wirkung auf den Tuberkelbazillus ausüben. Noch in einer Verdünnung von 1:2 Millionen wurde ein schädigender Effekt festgestellt. Auf Fochs Veranlassung hat dann Behring weitere Reagenzglasversuche mit dem Aurum kaliumcyanatum vorgenommen und die enorme Desinfektionskraft gegenüber Milzbrandbazillen geprüft. Es schien mir daher von großer Wichtigkeit, an ein Studium dieser Verbindung, die seitdem keine Beachtung mehr gefunden hatte, insbesondere in bezug auf ihre etwaige therapeutische Wirkung anf Tuberkulose heranzugehen. Der Gedankengang war dabei der, daß es zwar wohl nicht gelingen würde, mit Hilfe des Mittels die Tuberkelbazillen im Tierkörper abzutöten, aber es bestand in Anbetracht der enormen Wirksamkeit des Präparates die Hoffnung, vielleicht mit erträglichen nicht giftig wirkenden Dosen eine derartige Schädigung der $\mathrm{E}^{-}$ reger zu erreichen, daf dadurch eine therapeutische Wirkung erzielt wird.

Nach den bisherigen gemeinsam mit $D r$. Glück ausgeführten Untersuchungen und Tierversuchen hat nun das Mittel bei direkter Einführung in die Blutbahn beim Menschen einen auffallenden Heiteffelt bei Tuberlalose der Haut erzielt, ohne daß Schädigungen des Organismus auftreten. Wie weit diese Wirkung auszunutzen sein. wird, und wie sich das Präparat bei anderen Tuberkuloseformen bewährt, müssen weitere Prüfungen zeigen. Jedenfalls ist die bedeutungsvolle Tatsache festgestellt, daß es möglich ist, mit chemischen Substanzen vom Blutwege aus tuberkulöse Herde anzugreifen. Es wird die Aufgabe unserer weiteren Forsehung sein, ob sich vielleicht noch wirksamere und ungiftige Verbindungen finden lassen. Hoffen wir, daß es auf diesem Wege gelingt, zu einer neuen. Waffe im Kampfe gegen einen der furchtbarsten Feinde der Menschheit zu gelangen.

\section{Über das Verhältnis der Zellmechanik zur Entwicklungsmechanik.}

Vortrag, gehalten auf dem Dundee-Meeting der ,British Association for the advancement of science am 6. September 1912

Von Ludwig Rhumbler, Hann.-Münden.

Wilhelm Roux hat das Ziel der Entwicklungsmechanik in ein Stichwort zusammengefaßt; es heibt die mechanistische Erklärung der Entwicklung; dabei sind die Ausdrücke "Mechanik“ und ,mechanistisch" im allgemeinsten philosophischen Sinne der Lehre vom mechanistischen, das heiBt der Kausalität unterworfenem, Gesehehen gemeint. Die Entwicklungsmechanik hat demnach 